\title{
Gene expression profiling of orbital muscles in treatment-resistant ophthalmoplegic myasthenia gravis
}

Tarin A. Europa ${ }^{1,2}$, Melissa Nel ${ }^{1,2}$ and Jeannine M. Heckmann ${ }^{1,2^{*}}$ (D)

\begin{abstract}
Background: Unbiased in silico approaches applied to genome-wide data prioritized putative functional gene variants associating with treatment-resistant ophthalmoplegic myasthenia gravis (OP-MG). Although altered expression of genes harbouring these variants, or associated pathways, were shown in patient-derived transdifferentiated-myocyte models, gene expression in orbital-derived muscle was required to test the validity of the predictions.

Methods: We sampled orbicularis oculi muscle (OOM) and one paralysed extraocular muscle (EOM) from six individuals with OP-MG during blepharoptosis and re-alignment surgeries, respectively. For controls, the OOMs were sampled from four individuals without myasthenia undergoing surgery for non-muscle causes of ptosis, and one non-paralysed EOM. Using a qPCR array, expression of 120 genes was compared between OP-MG and control OOMs, profiling putative "OP-MG" genes, genes in related biological pathways and genes reported to be dysregulated in MG cases or experimental MG models, and in EOMs of cases with strabismus. Normalization was performed with two stable reference genes. Differential gene expression was compared between OP-MG and control samples using the $\triangle \Delta C T$ method. Co-expression was analysed by pairwise correlation of gene transcripts to infer expression networks.

Results: Overall, transcript levels were similar in OOMs and EOMs ( $p=0.72$ ). In OOMs, significant downregulated expression of eight genes was observed in OP-MG cases compared with controls ( $>$ twofold; $p \leq 0.016$ ), including TFAM, a mitochondrial transcription factor, and genes related to the following pathways: atrophy signalling; muscle regeneration and contraction; glycogen synthesis; and extracellular matrix remodelling. Several microRNAs, known to be highly expressed in EOMs, are predicted to regulate some of these genes. Co-expression analyses of genepairs suggested high interconnectedness of gene expression networks in OP-MG muscle, but not controls $(r>0.96$, $p<0.01$ ). Significant inverse directions of gene-pair correlations were noted in OP-MG versus controls OOM networks $(r \geq 0.92, p<0.001)$ involving most OP-MG genes overlapping prominently with muscle atrophy/contractility and oxidative metabolism genes.
\end{abstract}

Conclusions: The gene expression in orbital muscles derived from OP-MG individuals compared with normal controls, support the pathogenic hypothesis previously generated from whole genome sequence analyses. Repression of gene transcripts in OP-MG orbital muscle implicate tissue-specific regulatory mechanisms, which may inform future biomarker discovery approaches.

\footnotetext{
*Correspondence: jeanine.heckmann@uct.ac.za; jmheckmann01@gmail.com

${ }^{1}$ Neurology Research Group, Division of Neurology, Department

of Medicine, Faculty of Health Sciences, E8-74, New Groote Schuur

Hospital, University of Cape Town, Cape Town 7925, South Africa

Full list of author information is available at the end of the article
}

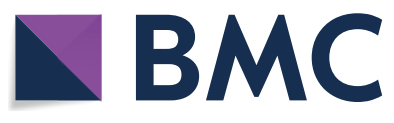

(c) The Author(s) 2020. Open Access This article is licensed under a Creative Commons Attribution 4.0 International License, which permits use, sharing, adaptation, distribution and reproduction in any medium or format, as long as you give appropriate credit to the original author(s) and the source, provide a link to the Creative Commons licence, and indicate if changes were made. The images or other third party material in this article are included in the article's Creative Commons licence, unless indicated otherwise in a credit line to the material. If material is not included in the article's Creative Commons licence and your intended use is not permitted by statutory regulation or exceeds the permitted use, you will need to obtain permission directly from the copyright holder. To view a copy of this licence, visit http://creativecommons.org/licenses/by/4.0/. The Creative Commons Public Domain Dedication waiver (http://creativeco mmons.org/publicdomain/zero/1.0/) applies to the data made available in this article, unless otherwise stated in a credit line to the data. 
Keywords: Orbicularis oculi, Muscle atrophy, Oxidative metabolism, Ophthalmoplegia, Extraocular muscles, Contractility, Myasthenia gravis, Treatment-resistant, Gene expression

\section{Introduction}

Previously we identified individuals with otherwise characteristic myasthenia gravis (MG) and who responded to immune therapies as expected in their non-ocular muscles, but who remained with treatment-resistant ophthalmoplegia with/without ptosis $[1,2]$. These individuals are most frequently MG subjects with juvenile onset symptoms, African genetic ancestry (see methods) and who have generalised disease with detectable circulating acetylcholine receptor $(\mathrm{AChR})$ antibodies $[1,3]$. Clinically, the treatment-resistant weakness of the extraocular muscles (EOMs) may range from moderate to severe. When severe, in which case most of the EOMs are paralysed, we refer to the subphenotype of treatment-resistant ophthalmoplegic MG, or OP-MG. In an African setting we anticipate up to $20 \%$ of cases may develop treatment-resistant EOM weakness [4].

To understand the underlying genetic pathogenesis of the OP-MG subphenotype we previously used next generation sequencing to dissect the molecular genetic landscape using an extreme phenotype approach i.e. OP-MG cases vs control MG. Genes associated with OP-MG were identified using single variant and gene-based cumulative variant statistical association analyses $[5,6]$. The pathogenetic pathways postulated to be altered in OP-MG, and supported by expression studies in patient-specific dermal-derived transdifferentiated myocyte models, related to muscle regeneration and atrophy signalling [7]. The aim of this work was to investigate the expression of these putative OP-MG susceptibility genes/pathways in tissues relevant to the subphenotype, i.e. patient-derived orbital muscles, and to verify the hypotheses generated by the unbiased genomic studies in the tissues of OP-MG cases vs controls without MG.

Briefly, a genome-wide single nucleotide variant analysis followed by prioritization based on skeletal muscle gene expression (from GTExPortal) [8], identified putative modifier variants in FAM92A1 and PEF1, which were more frequent in OP-MG vs control MG genomes $\left(p<1 \times 10^{-5}\right)$ [6]. As an adjunct, we sought genes with a cumulative variant burden at gene-level associating with OP-MG compared to control MG genomes, and after filtering by relevance in muscle (GTExPortal), identified several genes which may be relevant in OP-MG pathogenesis [6]. For simplicity we will refer to genes from both single-variant and gene-based analyses as putative "OP-MG genes" (Fig. 1, OP-MG $\mathrm{WGS}_{\mathrm{WG}}$ genes). Although the aim of the limb muscle-based prioritization was to select genes with biological relevance in this tissue, a limitation of this approach was that gene expression in EOM, as an unique muscle group, was not specifically considered due to the limited availability of gene expression data.

The EOMs constitute a unique muscle allotype [9-11]. However, we have used biopsies of orbicularis oculi muscle (OOM) as a surrogate orbital muscle to profile transcripts which may closely resemble the EOM transcriptome. We have done so for several reasons; the relative inaccessibility of EOMs; the ultra-rare indications to offer oculoplastic surgery in MG and therefore the possibility of EOM biopsy; and the paralysed EOMs in OP-MG would reflect the gene expression profile of this 'burnt-out' stage which may differ from the active evolving pathophysiological process.

As craniofacial muscles, both EOMs and OOMs share similar embryological origins and many of the anatomical, physiological and molecular characteristics which distinguish EOMs from skeletal limb muscle [12]. Both EOMs and OOMs have fast firing rates [13], high mitochondrial content and oxidative capacities $[14,15]$ and type II fibre predominance $[9,12]$. Therefore, in contrast to previous work using transdifferentiated cell models which originated from dermal fibroblasts, the use of OOMs as a surrogate for EOM will provide important validation of gene expression tissue-specificity of the craniofacial myotranscriptome in OP-MG vs normal controls.

\section{Methods}

\section{Patient selection and tissue collection}

OP-MG cases and controls selected for ocular re-alignment or lid elevation procedures were invited to participate and provided biopsy specimens with informed consent. The diagnostic criteria of OP-MG cases have been previously described $[1,6,7]$, and those included in this report all had circulating anti-acetylcholine receptor $(\mathrm{AChR})$ antibodies $(>0.4 \mathrm{nmol} / \mathrm{L})$ (Table 1$)$. Importantly, for OP-MG patients to be eligible for ptosis surgeries (lid elevation), OOM contractility had to be sufficient to minimize the risk of post-operative corneal exposure. In contrast, the EOMs of the OP-MG case had been paralysed for several years by the time the medial rectus was sampled. Control samples were derived from cases with strabismus or ptosis from non-MG causes. All subjects had self-reported African-genetic ancestry i.e. either black African genetic ancestry, or mixed-African ancestry comprising 


\begin{tabular}{|c|c|}
\hline Category & Genes \\
\hline $\begin{array}{l}\text { OP-MG genes } \\
\text { WGS }^{1}\end{array}$ & $\begin{array}{c}\text { FAM92A1, PEF1, MKNK2, AKT1S1, SH3BGR, MYL12B, } \\
\text { PPP1R12C, PPP1R2, ZFP36L2 }\end{array}$ \\
\hline $\begin{array}{l}\text { WES }{ }^{2} \\
\text { OP-MG pathways } \\
\text { Muscle regeneration }\end{array}$ & $\begin{array}{c}\text { IL6R, SPTLC3, DDX17, ST8SIA1, FAM136A, FAM69A, CANX, } \\
\text { PPP6R2, HLADPB1 }\end{array}$ \\
\hline $\begin{array}{r}\text { - gangliosphingolipid } \\
\text { synthesis }^{2}\end{array}$ & SPTLC1, UGCG \\
\hline - satellite cell activation 3 & HGF, PAX6, PAX7, PAX3 \\
\hline - myogenesis $4,5,6$ & MYOG, TGFB1, BMP4, MSTN, TWIST1, WNT3, WIF1 \\
\hline Atrophy signalling ${ }^{7}$ & \\
\hline -FOXO pathway & $\begin{array}{c}\text { TRIM63 (MuRF1), FBXO32 (atrogin-1), FOXO3, TNF, IL6, } \\
\text { MAPK6, HDAC1, SIRT1 }\end{array}$ \\
\hline -IGF-1 pathway & IGF1, PIK3CB, PIK3R1, AKT1, AKT2, MTOR \\
\hline -Proteolysis (UPS) & BAX, BAK1, TMBIM6, BCL2, CASP3, CASP6, CAPN3, XIAP \\
\hline $\begin{array}{l}\text { Mitochondrial biogenesis/ } \\
\text { mitophagy }^{7}\end{array}$ & $\begin{array}{c}\text { PPARD, PPARG, NRF1, NRF2, TFAM, TFB1M, TFB2M, CRLS1, } \\
\text { DNM1L, OPA1, MFN2, ESRRA, ESRRG, CAMK2G, BNIP3, } \\
\text { PINK1, PARK2 }\end{array}$ \\
\hline MG/ EAMG pathways & \\
\hline AChR and MuSK & CHRNA1, CHRNG, CHRNE, MUSK \\
\hline Inflammatory response 8,9 & $\begin{array}{c}\text { RGS2, NFKBIA, NFIX, ERRFI1, GLIPR2, DYRK2, } \\
\text { GALNT12, ANKRD1, GPNMB, CEBPD, DDIT4, GADD45A, } \\
\text { MT1A, FKBP5, CD55 }\end{array}$ \\
\hline Oxidative metabolism ${ }^{8,9}$ & FMO2, ENC1, UCP3, PDK4, ANGPTL4, ACSL5 \\
\hline Strabismus pathways & \\
\hline Muscle regeneration ${ }^{10,11}$ & $\begin{array}{c}\text { GDNF, IGF1R, CXCR4, PPARGC1A, IL1ORA, NOTCH2, } \\
\text { MYH1, MYH2, MYH3, MYH13 }\end{array}$ \\
\hline $\begin{array}{l}\text { Extracellular matrix } \\
\text { remodelling10,11 }\end{array}$ & $\begin{array}{c}\text { COL1A1, COL8A1, CTGF, CYR61, LOX, MMP16, MMP3, TIMP4, } \\
\text { TNC, VCAN, DDR2, MMP2, ANK1, DES, NRCAM }\end{array}$ \\
\hline Reference genes $9,10,12,13$ & RPLPO, ACTB, GAPDH, CSNK2A2, ACTN2 \\
\hline
\end{tabular}

Fig. 1 Genes profiled in the array organised by category. Ophthalmoplegic myasthenia gravis (OP-MG). "OP-MG genes" refers to genes identified by unbiased next generation sequencing analyses in OP-MG and control-MG subjects, either by whole genome sequencing (WGS) or whole exome sequencing (WES). "OP-MG pathways" refers to the main biological pathways associated with the known physiological functions of the putative OP-MG genes. The "atrophy signalling" genes were selected from biological pathways in reference 7 using associated KEGG pathways as resources. Ubiquitin proteasome system (UPS); experimental autoimmune myasthenia gravis (EAMG); acetylcholine receptor (AChR); muscle-specific kinase (MuSK); extracellular matrix (ECM). "Strabismus pathways" refers to genes with altered expression in human strabismic extraocular muscles. References in figure: (1) [5]; (2) [6]; (3) [50]; (4) [51]; (5) [12]; (6) [52]; (7) [32]; (8) [53]; (9) [18]; (10) [21]; (11) [22]; (12) [7]; (13) [54]

predominantly KhoiSan ancestry, with lesser genetic contributions from black Africans, Europeans and Asians (see [6]). The study was approved by the institutional Human Research Ethics Committee (HREC 257/2012).
Biopsies of the orbital part of the orbicularis oculi $(\mathrm{OOM})$ were received from those undergoing lid elevation procedures and medial recti muscle samples (EOM) from ocular realignment surgery. Both OOM and EOM samples were dissected by the ophthalmic surgeon 
Table 1 Clinical information of patient-derived orbicularis oculi muscle and medial recti samples

\begin{tabular}{llll}
\hline $\begin{array}{l}\text { Age } \\
\text { at surgery } \\
\text { (years) }\end{array}$ & Orbital muscle & Diagnosis & $\begin{array}{l}\text { MG } \\
\text { duration } \\
\text { (years) }\end{array}$ \\
\hline 42 & OOM & OP-MG & 18 \\
15 & OOM $^{\text {a }}$ & OP-MG & 7 \\
27 & OOM & OP-MG & 10 \\
34 & OOM & OP-MG & 14 \\
55 & OOM & Levator dehiscence & NA \\
51 & OOM & Levator traumab & NA \\
56 & OOM & Canalicular obstruction & NA \\
27 & EOM & OP-MG & 15 \\
77 & EOM & Secondary esotropia- & NA \\
& & blind from glaucoma & \\
\hline
\end{tabular}

Myasthenia gravis (MG) duration was taken from onset of symptoms. Extraocular muscle (EOM) refers to medial rectus; ophthalmoplegic MG (OP-MG); orbicularis oculi muscle (OOM)

a Samples obtained from both eyes at independent surgeries

b Normal OOM biopsied

without cautery or clamping, immediately placed in RNAlater (ThermoFisher) and stored at $-80^{\circ} \mathrm{C}$.

\section{RNA isolation}

The orbital muscle samples were homogenized in TRI Reagent (ThermoFisher) using a handheld Pellet Pestle (Fisherbrand). After chloroform precipitation, RNA was isolated from the aqueous phase using a spin-column method (Direct-zol Miniprep kit, Zymo) according to the protocol which included an in-column DNAse step. RNA was quantified using Nanodrop 1000 software (v3.5.2, Inqaba Biotechnical industries) and RNA integrity (RIN) was evaluated using the PicoChip Bioanalyzer (Agilent 2100 Bioanalyzer). The muscle samples weighed 3-26 mg yielding 5-49 ng/ $\mu \mathrm{L}$ RNA. Spectrophometric ratios suggested some impurities despite optimised RNA extraction protocols using phenol/chloroform precipitation, but were in the expected range for RNA isolated from muscle [16] with A260/280 range 1.6-1.9 and A260/230 range 0.4-1.6. RIN values ranged between 5.2 and 7.6.

\section{Custom array plate}

A total of 120 target and 5 reference genes were profiled in a custom qPCR array (Qiagen) using proprietary assays. The target genes were categorized into OP-MG genes, OP-MG pathways, MG pathways and strabismus pathways based on the source of evidence for their relationship to OP-MG, MG or strabismus, although there is overlap between categories (Fig. 1). OP-MG genes broadly refer to those identified by WGS [6] and the preceding whole exome sequencing (WES) analysis [5], although the prime objective of this study was to validate the genes identified by the WGS analysis. OP-MG genes also served as "seed queries" which informed the broader enquiry of additional gene candidates in related pathways (OP-MG pathways). Genes previously shown to be dysregulated by MG in human limb muscles [17], in vitro models or experimental autoimmune myasthenia gravis (EAMG) [18] were also included. As reduced contractility (as occurs in OP-MG EOMs) [1,19] potentially alters the regulation of biological pathways in ocular muscle, we also profiled genes previously shown to be dysregulated in the EOMs of cases with ocular misalignment/ strabismus due to other non-myasthenic causes. We postulated that functional variants in muscle contractility pathways may render EOMs vulnerable in the setting of MG weakness.

The reference genes were selected from genes that have previously been validated in human $[9,20-22]$ and rodent EOMs $[18,23]$ and showed stability in a patient derived muscle model [7].

\section{Reverse transcription, qPCR and normalization}

For each muscle RNA sample 40 ng was reverse transcribed using the $\mathrm{RT}^{2}$ First Strand Kit (Qiagen) according to the manufacturer's protocol and qPCR performed using the $\mathrm{RT}^{2}$ SYBR Green Mastermix (Qiagen) on the QuantStudio 12K Flex thermocycler. No inhibition of reverse transcription or PCR was evident and no genomic DNA contamination was detected. Three control EOM samples with the lowest RNA concentrations and RIN values were excluded from data analysis for quantitation cycle $(\mathrm{Cq})$ values $>35$ for at least $15 \%$ of genes in the array (see Additional file 1: Table S1). Reference genes for normalization were selected based on the geNorm [24] and Bestkeeper methods for OOMs [25] and chosen based on stability of expression levels for the two EOMs.

\section{Gene expression analysis and statistical methods}

Raw Cq values from the array were analysed using the $\Delta \Delta \mathrm{Ct}$ method [26]. Differences between OP-MG and controls were expressed as fold change or $\log _{2}$ fold change (Prism Graphpad 8). For OP-MG vs control comparisons in OOMs, unpaired Student's $t$ tests for normally distributed $2^{-\Delta \mathrm{Cq}}$ values and Mann-Whitney $\mathrm{U}$ tests for non-parametric data, were performed. Shapiro Wilk tests were performed to assess normality. The Benjamini-Hochberg correction was performed on the results of the $t$ tests and Mann-Whitney $U$ tests in the OOM comparisons. Due to the small sample, the false discovery rate (FDR) correction was set at $15 \%$. Statistical significance of the difference in gene expression by phenotype could not be calculated for EOMs. 
Using OOM data, pairwise gene co-expression by phenotype was performed by Pearson's correlations. Genes not expressed in $>1$ sample $(\mathrm{Cq}>35)$ were excluded from these analyses $(n=5)$. Correlation plots using gene pairs with significant correlations $(p<0.01)$ were generated using the Corrplot $\mathrm{R}$ package. Hierarchical clustering of the OP-MG correlation plot was performed using Hmisc (R package) and the controls correlation plot was reordered to match for visual comparison. Co-expression networks were generated using Cytoscape (v.3.71). Potentially functional modules were identified using the MCODE Cytoscape plug-in. To determine which gene pairs were significantly different between OP-MG and controls, differential correlation analysis was performed using the DiffCorr R package [27]. Gene pairs with a local false discovery rate $>0.6$ were excluded.

\section{Results}

Six OP-MG muscle (1 EOM; 5 OOM) and five control muscle (1 EOM; 4 OOM) samples passed data quality analysis (Table 1 ).

\section{Differential gene expression in orbicularis oculi muscles}

To assess differences in relative gene expression between 5 OP-MG and 4 control OOMs, data normalization was performed using the average values for RPLPO and ACTN2 as reference genes (Additional file 1: Table S2).

Figure 2 shows the genes that showed $>$ twofold difference in gene expression levels between OP-MG vs control OOMs $(\mathrm{FDR}=15 \% ; p<0.01)$. All the significantly differentially expressed genes were downregulated in the OP-MG muscles. Interestingly, the three most differentially repressed gene transcripts in the OP-MG samples were those genes included in the profiling array because of their altered expression levels reported in post-mortem strabismic EOMs compared with controls [21]. $M Y H 2$, which encodes the myosin heavy chain 2a isoform, found in fast-twitch muscle fibres and abundant in both OOMs [13] and EOMs [28], showed fivefold downregulation compared to controls $(p=0.008) . V C A N$ and $A N K 1$, genes related to extracellular matrix remodelling pathways, as well as genes related to muscle atrophy signalling, CAPN3 and MAPK6 $(p<0.009)$ were downregulated in the OP-MG OOMs. VCAN and ANK1 encode components of the extracellular matrix and muscle, respectively, that regulate myoblast fusion/differentiation and attachment to the sarcoplasmic reticulum in regenerating muscle [29]. CAPN3 (calpain 3) is involved in preserving sarcomeric integrity [30]. MAPK6/ERK3 encodes mitogen-activated protein kinase- 6 , and acts at integration points for multiple processes including myogenesis [31]. TFAM, encoding a critical mitochondrial DNA

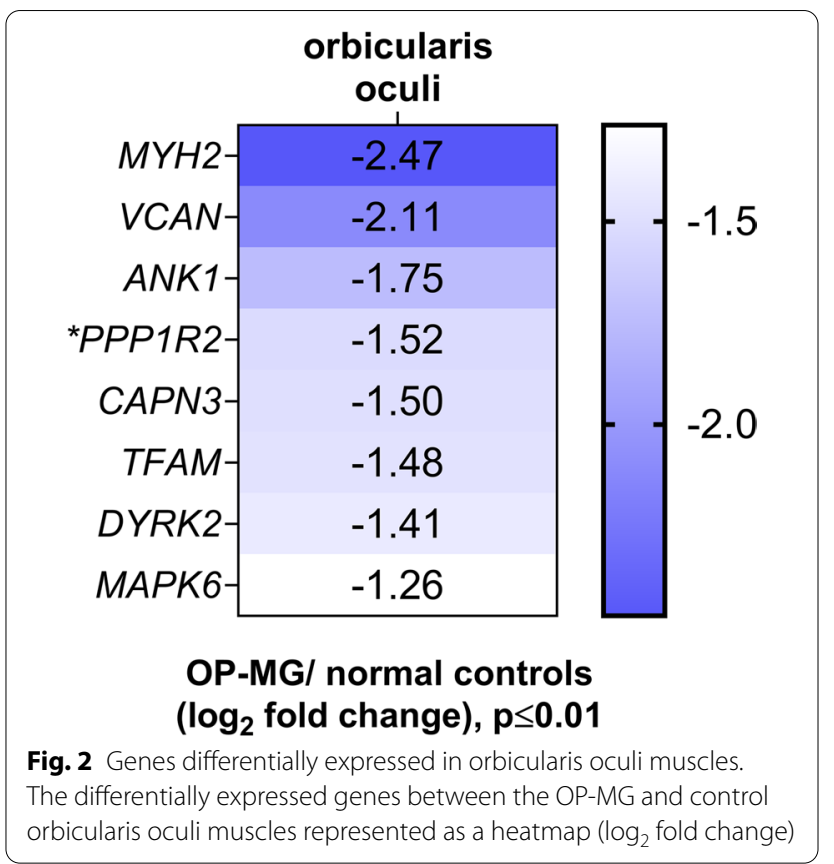

transcription factor [32], was differentially downregulated $(p=0.005)$ in OP-MG.

PPP1R2 which was prioritized as an OP-MG gene and showed significant repression in OP-MG orbital muscles (2.9-fold; $p=0.016$ ), encodes a key enzyme in glycogen synthesis, phosphatase-1 regulatory inhibitor subunit R-2. The repression of $P P P 1 R 2$ transcripts together with DYRK2 (2.7-fold; $p=0.003)$, which phosphorylates glycogen synthase, suggest dysregulation of muscle energy metabolism in OP-MG muscles, but not control muscle.

Overall, we found differential repression of genes involved in the maintenance of the extracellular matrix and sarcomeric stability, as well as mitochondrial biogenesis and muscle metabolism in orbital muscles from OP-MG cases vs normal controls. Interestingly, genes in the latter categories were included in the array based on postulated pathway involvement informed by genomic studies, whereas the former were considered "strabismuspathway" genes at the time and included as a controls for the clinical phenotype of altered EOMs contractility in OP-MG.

\section{Differential gene-co-expression in orbicularis oculi muscle by phenotype}

Pairwise correlations identified 232 strongly correlated gene pairs in OP-MG OOMs and 92 gene pairs in controls $(\mathrm{r}>0.96, p<0.01)$. OP-MG and control correlation plots were generated using this data and hierarchical clustering ( $k$-median) identified potentially functional modules [33] visualised in the OP-MG plot that were 
not seen in the control plot (Fig. 3a vs b). Figure 3c shows the visual network of co-expressed gene pairs in the OOMs of OP-MG samples (from Fig. 3a) and the most interconnected genes (10-15 gene-gene interactions each) involved putative OP-MG genes from the WGS analysis (FAM92A1) and from the WES analysis (CANX, DDX17, FAM69A) (Fig. 3c; unlabelled OP-MG genes are from the WES analysis). DDX17 (Fig. 3c solid arrow) is a master transcriptional regulator involved in muscle gene expression [34] and appears central to a subnode. CANX encodes calnexin, a chaperone protein that facilitates the assembly of $\mathrm{AChR}$ subunits [35], FAM92A1 has recently been recognized for its importance in mitochondrial functioning [36] and both FAM69A and PEF1 have poorly characterized biological function. PEF1 encoding Peflin, which may be involved with endoplasmic reticulum-golgi transport and/or calcium binding [37], connected with a 'module' of genes (Table 2) which were profiled because they showed altered muscle expression levels in EAMG [18], and they are known to regulate mitochondrial biogenesis and oxidative metabolism. PARK2, encoding parkin, a major regulator of mitophagy [32], appeared to form a subnode of negatively correlated genes (Fig. 3c, dashed arrow), several of which are involved in muscle regeneration (e.g. $D D X 17$ ) and mitochondrial biogenesis. PPP1R2, which was differentially repressed in OP-MG muscle (Fig. 2), did not correlate with other transcripts

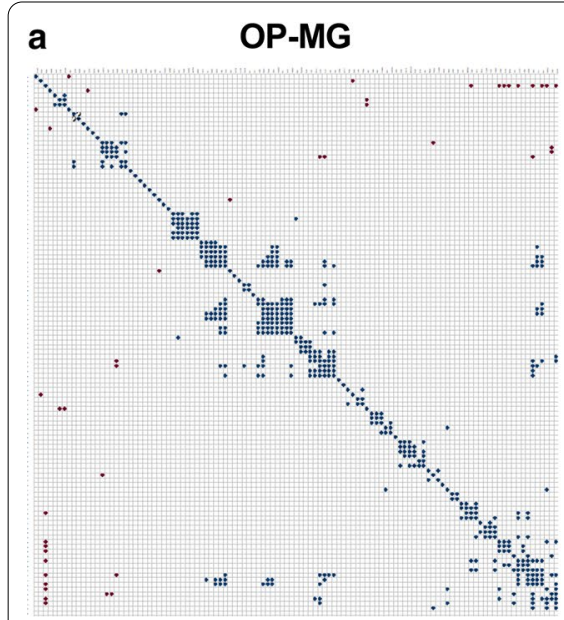

\section{c}
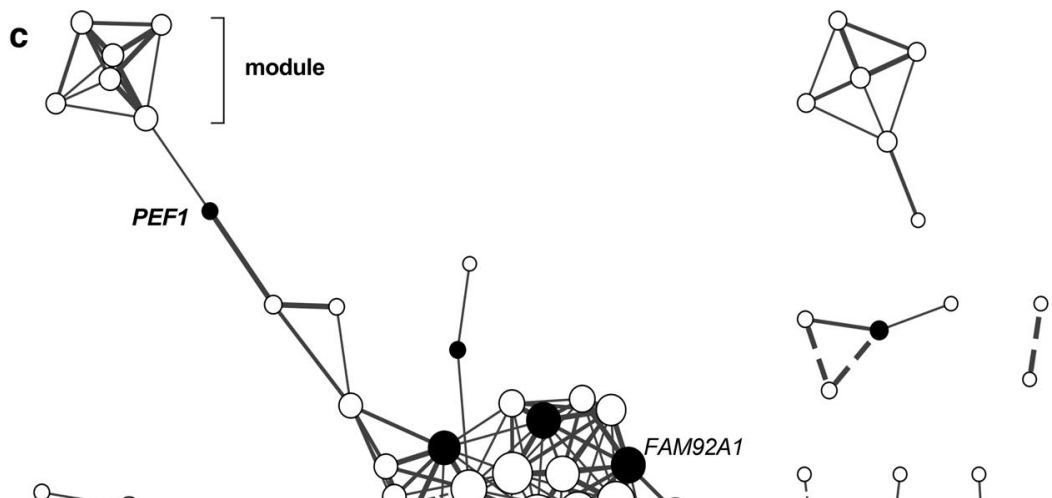

b

controls
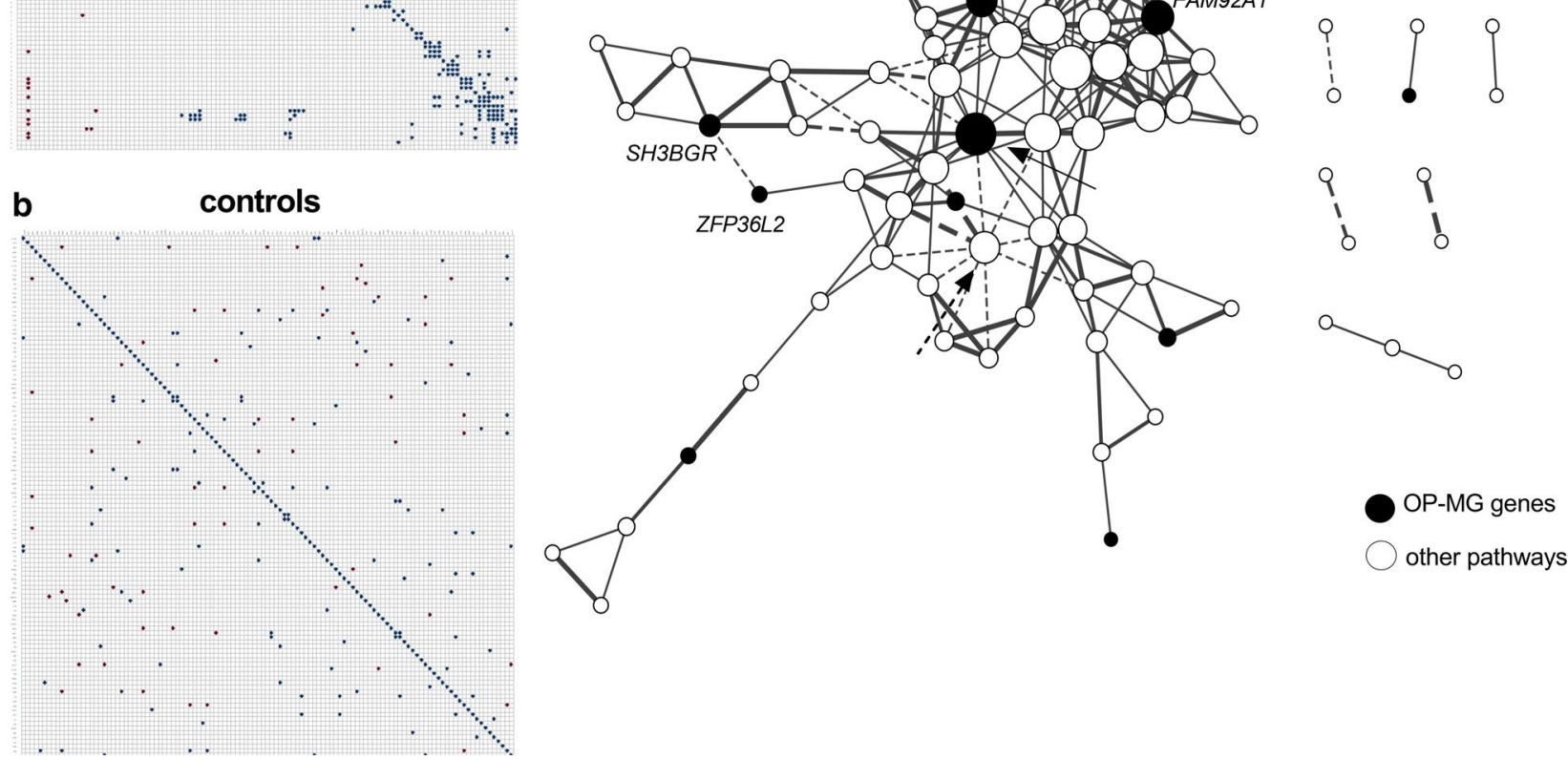

Fig. 3 Visual comparison of gene co-expression between OP-MG and control orbicularis oculi muscles. a Ophthalmoplegic myasthenia gravis (OP-MG) orbicularis oculi muscles (OOMs) correlation plot with gene pairs $(r>0.96 ; p<0.01)$. b Control correlation matrix $(r>0.98 ; p<0.01)$ ordered to match hierarchical clustering configuration of A for comparison. c Gene co-expression network of OP-MG orbicularis oculi muscles ( $r>0.96$; $p<0.01$ ); the OP-MG genes, identified by whole genome sequencing analysis, are labelled (intermodular gene in bold). Degree of interconnectivity is shown by increased node size. Interconnecting lines are weighted by strength of correlation. Dashed lines indicate negative correlations. The solid arrow indicates DDX17, which is central to a subnode and the most highly prioritized gene from the whole exome sequencing analysis [5]. The dashed arrow indicates PARK2 which is central to an inhibitory subnode 
Table 2 Genes in putative co-expression network with PEF1 transcripts in OP-MG orbicularis oculi muscle

\begin{tabular}{ll}
\hline Gene & Function (biological pathway) \\
\hline ANGPTL4 & Oxidative metabolism \\
PPARG & Mitochondrial biogenesis/metabolism \\
FMO2 & Oxidative metabolism \\
BCL2 & Mitochondrial biogenesis/metabolism \\
TIMP4 & $\begin{array}{c}\text { Inhibitor of metalloproteinases (extracellular matrix remod- } \\
\text { elling) }\end{array}$ \\
MMP16 & Metalloproteinase (extracellular matrix remodelling) \\
\hline
\end{tabular}

in OP-MG muscle yet showed significant correlations with four genes in control OOMs (data not shown).

Next, a differential co-expression algorithm was used to statistically identify gene pairs differing significantly and inversely between the two phenotypes (Fisher's $\mathrm{Z}$ tests, $p<0.002)$. An example is showed in Fig. 4 a with significant positive correlations in a gene pair in one phenotype (OP-MG or controls) but inverse correlations in the other $(p<0.01)$ (see Additional file 1: Figure S1). Figure 4b displays a correlation network of the significant inversely correlated gene pairs for the OP-MG network (implying that the control network showed opposite correlations).

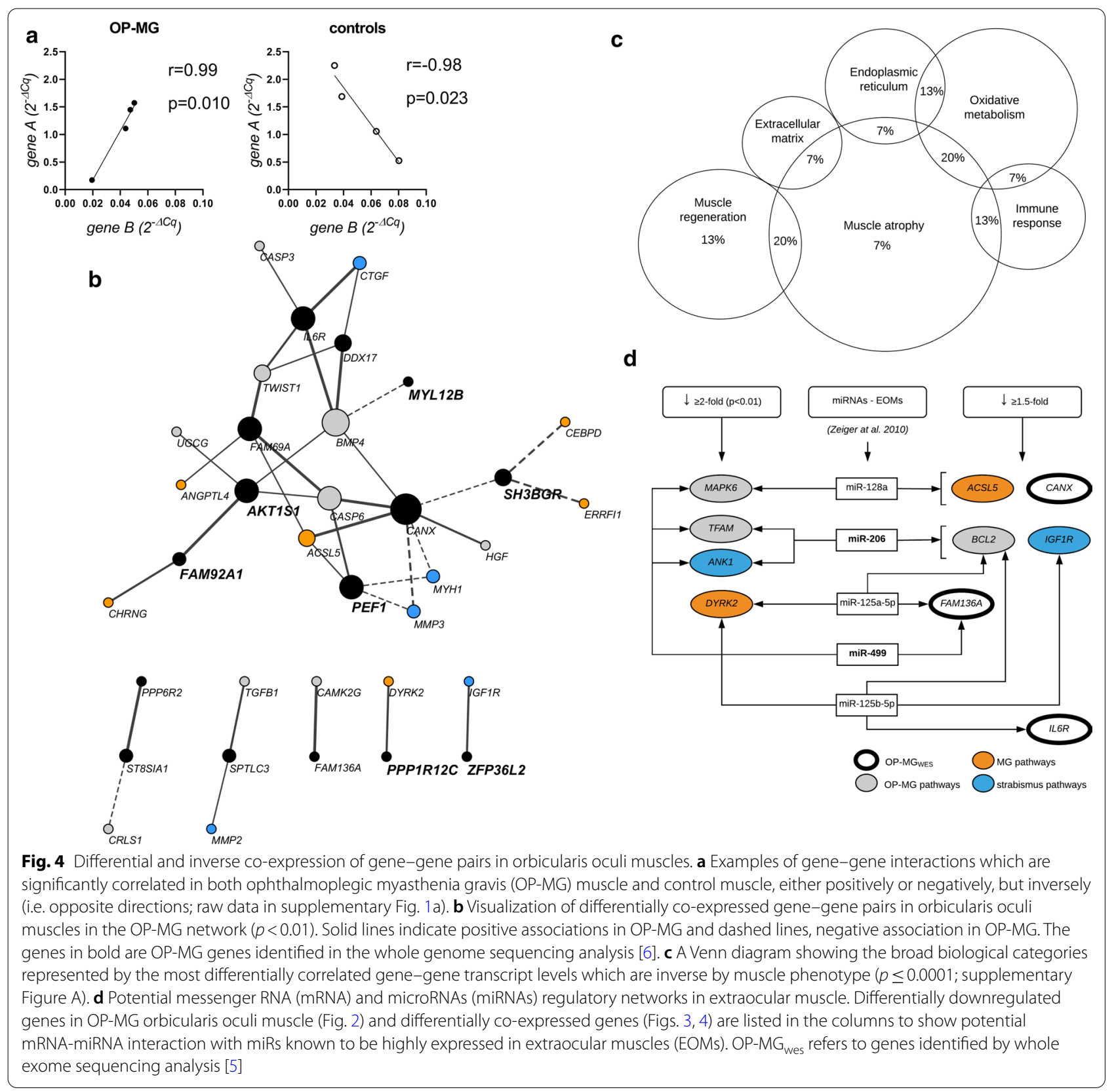


The importance of this analysis is that it shows inverse gene-gene interconnectivity (correlations) in OP-MG orbital muscle vs controls, which included seven (of nine) genes prioritized as OP-MG genes in the previous unbiased genomic comparisons viz. PEF1, FAM92A1, AKT1, $M Y L 12 B, P P P 1 R 12 C, Z F P 36 L 2$ and SH3BGR [6]. Interestingly, the gene product of $S H 3 B G R$ is involved with myosin heavy chain isoforms and sarcomere function and is highly expressed in EOMs.

Figure 4c depicts the representative biological categories of strongly correlated gene pairs in the OP-MG network and inverse in controls. Of the most differentially correlated gene pairs which were inverse by phenotype $(\mathrm{n}=13 ; p \leq 0.001),>50 \%$ involved a gene whose transcripts were substantially altered in EAMG [17] suggesting altered MG pathways in the muscle transcriptome of OP-MG vs controls. The broad category of 'muscle atrophy' signalling was the most representative functional category accounting for two-thirds of the most differential and inversely correlated gene pairs by phenotype with oxidative metabolism the next most frequent category ( $p \leq 0.001)$ (genes may be implicated in 2 categories).

\section{Downregulated genes in OP-MG orbital muscle may be regulated by microRNAs}

In OOMs the significant differential downregulation of transcripts in OP-MG suggest post-transcriptional gene repression by microRNAs (miRs) binding to $3^{\prime}$ regulatory variants in OP-MG as a potential regulatory mechanism. Therefore, the miRTarBase database [38] was interrogated for interactions between miRs previously shown to be highly expressed in EOMs [39], and genes differentially downregulated $\geq 1.5$-fold in OP-MG, compared with controls. MiR-499 and miR-206, the most highly expressed miRs in EOM (>sevenfold higher than in skeletal muscle), potentially interact with three of the genes which showed differential repression in OOMs (Fig. 4d). In addition, CANX and IL6R, which feature prominently in the differential gene-gene cross-correlations (Fig. 4b), and predicted to have EOM-miRs interactions, were prioritized previously to have $3^{\prime}$ regulatory region variants present in the OP-MG, but not controls $[5,7]$.

\section{Extraocular muscle}

Although only two EOMs (medical recti) passed quality control we briefly mention the results due to scarcity of data on these tissues from live donors. For the EOMs, $A C T B$ was used for normalization $(\mathrm{SD}=0.06$ between samples; Additional file 1: Table S2). Of relevance to the interpretation of the OOM results, the normalized expression levels of the 125 genes were similar between the two EOMs and nine OOMs $(p=0.72)$, although the EOM-specific isoform, $M Y H 3$, differed significantly $(p<0.0001$; Additional file 1: Table S3, Figure S2). As the comparison of gene expression between only two samples required cautious interpretation a quantile-quantile plot of the transcripts of the two samples showed a similar distribution of the majority of the genes with only isolated values $>$ tenfold difference (Fig. 5a, b). We briefly discuss WIF1, IL6 and MT1A transcript levels which were $\geq 25$-fold higher in OP-MG compared to control.

WIF1 encodes Wnt inhibitory factor-1, and as an inhibitor of Wnt signalling may affect muscle regeneration and
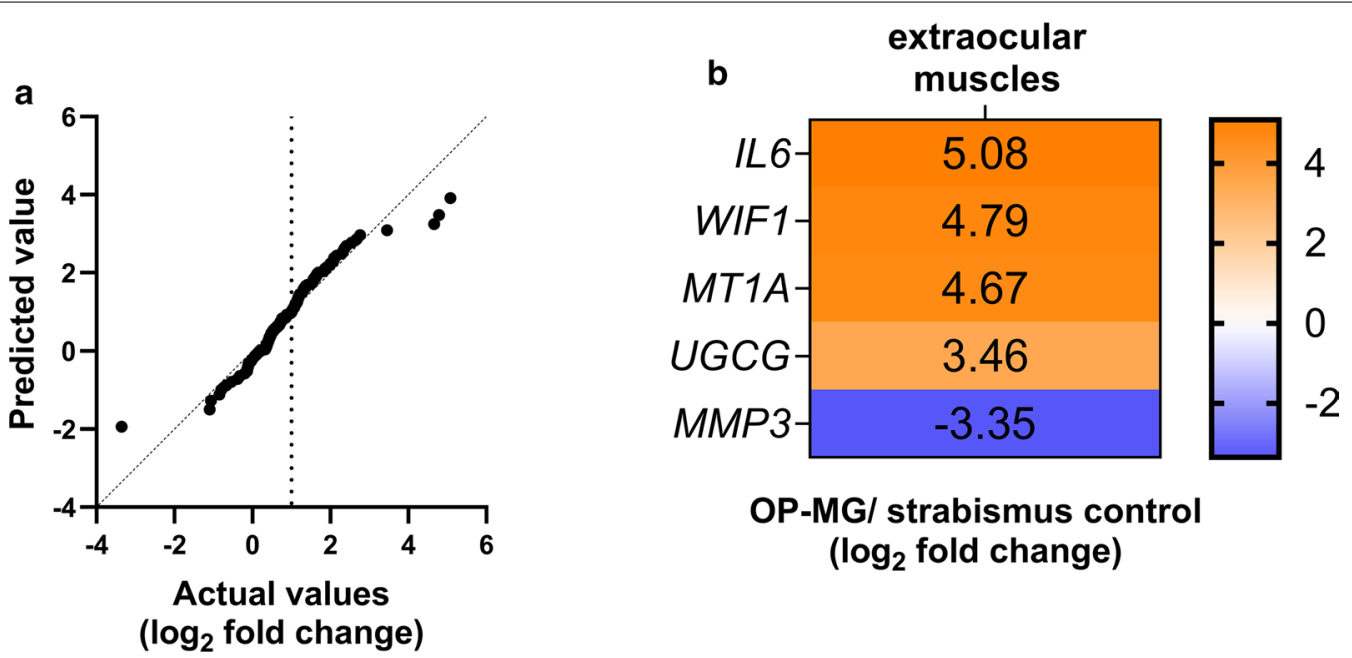

Fig. 5 Genes differentially expressed in the medial rectus of an ophthalmoplegic myasthenia gravis (OP-MG) patient. a Quantile-quantile plot showing the distribution of $\log _{2}$ fold change values between the OP-MG and control extraocular muscle (medial recti) and $\mathbf{b}$ outlying values with > tenfold differences in gene expression values are represented in the heatmap 
endplate AChR clustering [40]. MT1A encodes metallothionein-1A and protects cells against oxidative stress [in [18]]. Increased expression of metallothionein 1 has been reported in atrophic muscle, possibly by preventing the upregulation of the insulin growth factor (IGF)-1 pathway [41], and in the EOMs of the EAMG rodent models [18]. IL6 encodes interleukin-6, a pivotal myokine in satellite cell/myoblast proliferation amongst others, but with persistently elevated levels it facilitates muscle atrophy and inhibits IGF-1 signalling [42]. Therefore, the higher transcript levels of IL6 and MTIA in the chronically paralysed EOM of an OP-MG vs a non-paralytic control, could be compatible with severely atrophic muscle and/or MG-induced effects [17].

\section{Discussion}

Gene expression profiling in orbicularis oculi muscles showed the genes which were previously prioritized as OP-MG-associated variants, were differently regulated in the muscles of OP-MG cases compared with normal controls, and supported their involvement in OP-MG pathogenesis. The original gene discovery strategy used an 'extreme phenotype' in which all the cases had MG, but who differed only by their EOM resistance to treatment observed in the clinic. In the current study we aimed to validate expression differences in these putative OP-MG genes/pathways using orbital muscles from affected cases vs controls without MG. Differential gene repression and gene-gene correlations differed significantly between the OOMs from OP-MG cases and control muscle. Importantly, genes such as PEF1 and FAM92A1, which featured prominently in the dysregulated gene correlation networks by phenotype, were genes with unknown function when prioritized and included in the custom panel. FAM92A1, has recently been found to be critical for mitochondrial ultrastructural integrity [36]. Although PEF1 remains with poorly characterised function [43], the strong correlations with the transcripts of several genes related to oxidative metabolism and extracellular matrix remodelling, suggest "guilt-by-association". These findings are highly suggestive of perturbed crosstalk in the OP-MG orbital muscles between genes representing the muscle atrophy/regeneration and mitochondrial/oxidative metabolism functional categories.

In addition to assessing the putative OP-MG gene transcripts in orbital muscles, we were also interested in whether the transcriptional profile of genes known to represent MG/EAMG and strabismus-related pathways, may cross correlate with OP-MG gene transcripts. Here we included strabismus-related genes based on reports showing dysregulation in maligned EOMs (strabismus) in a non-MG context. However, recently RNAsequencing of cultured human myoblasts revealed that
AChR-antibodies significantly impacted genes related to extracellular matrix and actin/myosin cytoskeleton pathways [44], which overlap with the genes categorised in Fig. 1 as 'strabismus pathways', such as myosin genes. Although only selected myosin genes were included in the OOM profiling, they featured as dysregulated genes (Figs. 2, 4), and underscore the previous hypothesis based on clinical and histopathological reports in MG muscles, that loss of muscle contractility is critical in driving OP-MG pathogenesis $[19,45]$. These results support the postulate that the EOMs of susceptible individuals, carrying gene variants in the myosin genes/pathways amongst others, are particularly vulnerable during the active MG attack to extended periods of poor contractility.

Genes related to 'muscle atrophy signalling' pathways at cellular level including those regulating muscle fibre size by protein synthesis (IGF1-Akt/mTOR signalling pathway), degradation (ubiquitin-proteosome and autophagylysosome pathways) and the balance maintained by the TGF $\beta$-BMP superfamily [46], were common to differential gene-pair cross-correlations summarised in Fig. 4c. Interestingly, $M Y H 2$ which showed significant repression in the OP-MG OOMs, also featured prominently in the muscle genes regulated by AChR-antibodies [44]. MYH2 is a substrate of MuRF1 and therefore directly responsive to atrophy signalling [47]. MuRF1, in turn, is moderated by the IGF1/PI3K/Akt signalling pathway which inhibits induction of muscle atrophy pathways [47]. The observed gene-pair correlations, in which transcript levels in genes from these pathways in OP-MG muscle were in direct opposite to those observed in controls, suggest perturbations between the muscle atrophy, cytoskeletal components and regeneration pathways in OP-MG OOMs. A concern for the translation of these results to the clinic is that under experimental conditions using dexamethasone, myosin heavy chain transcripts are downregulated resulting in steroid-linked muscle atrophy [47]. Steroid therapy, such as prednisone, is an important adjunct to immune therapies in MG, and in the early management of myasthenic ocular muscle paralysis, we have observed improved clinical outcomes linked with higher doses of prednisone [4]. However, it is not known whether higher doses of prednisone will benefit all individuals with ocular muscle involvement such as those carrying functional variants in high-risk pathways $[6,48,49]$.

The differential gene downregulation of gene transcripts in OP-MG muscle compared to controls, implicate regulatory mechanisms mediated by miRs. Bioinformatic tools predict several miR-mRNA interactions by miRs which are highly expressed in EOMs and genes which showed differential repression in OP-MG muscle. Furthermore, OP-MG associated genes with putative functional $3^{\prime}$ regulatory variants, such as $D D X 17$ 
$[5,7]$ (Figs. 3c, 4b), featured prominently in the differential and inverse cross-correlations by phenotype in the orbital muscles, suggesting its involvement in dysregulated crosstalk. $D D X 17$, which was highly expressed in both EOMs and OOMs, is a master regulator in muscle splicing events and miRNA biogenesis [34]. The study of circulating miRs as possible prognostic biomarkers for treatment-responsiveness in EOMs, may prove useful for future clinical treatment trials.

We acknowledge a number of limitations to this work. The sample sizes are limited due to opportunistic sampling of rare events. As the opportunity to evaluate the expression of putative OP-MG genes in the EOM of a well-characterized OP-MG case is a rarity, even the comparison of a single OP-MG medial rectus sample to a control was considered a valuable addition despite years of paralysis in the OP-MG EOM which would have impacted the gene-transcript snapshot. Although the study conclusions are based on the expression levels within the larger sample of OOMs and despite the similar origins of EOMs and craniofacial muscles (orbital orbicularis oculi), the EOM allotype differs from OOMs in some respects notably fatigue resistance, multi-innervated fibres (compared to singly innervated fibres in OOMs) and EOMs are constantly regenerating $[12,15]$. Nevertheless, the orbital-derived orbicularis oculi muscles were not clinically weak and therefore a good comparator to the normal control muscle transcripts, and the gene transcript levels did not differ significantly between EOMs and OOMs.

\section{Conclusion}

The profiling of patient-derived orbital muscle supports the previous unbiased, genome-driven hypotheses of candidate genes and pathways involved in the pathogenesis of OP-MG. The dysregulated gene expression in the orbital muscles of treatment-resistant ophthalmoplegic MG cases implicate pathways related to muscle contractility and mitochondrial homeostasis and strongly suggests altered extraocular muscle-specific regulatory events.

\section{Supplementary Information}

The online version contains supplementary material available at https://doi. org/10.1186/s13023-020-01629-9.

Additional file 1. Supplementary Table S1 present the results of the quality of RNA extracted from the orbital muscles. Table S2 depicts the raw data informing the reference gene selection and Table $\$ 3$ the genes which were highly expressed in extraocular muscle and orbicular oculi muscles. Supplementary Figure S1. Differentially co-expressed gene pairs between OP-MG and control orbicularis oculi muscle ranked by significance derived from Fisher's Z test. Figure S2. Scatter plot of gene expression levels in two extraocular muscles and nine orbicularis oculi muscles.
Abbreviations

MG: Myasthenia gravis; OP-MG: Ophthalmoplegic myasthenia gravis; EOMs: Extraocular muscles; OOMs: Orbicularis oculi muscles; EAMG: Experimental autoimmune MG; AChR: Acetylcholine receptor; RIN: RNA integrity number; qPCR: Quantitative PCR; Cq: Quantitation cycle value; SD: Standard deviation; $C V$ : Coefficient of variation; $\triangle$ : Delta; FDR: False discovery rate.

\section{Acknowledgements}

The authors wish to thank Dr M Lenake and Prof A Murray from the University of Cape Town (UCT) Ophthalmology division for their assistance in obtaining the samples with such care, and to the patients who agreed to the muscle biopsies.

\section{Authors' contributions}

TAE generated, analyzed and interpreted the data and wrote the first draft. MN and JMH supervised the project and were both major contributors in editing the manuscript. All authors read and approved the final manuscript.

\section{Funding}

The National Research Foundation (NRF) of South Africa provided funding (113416, 109084, JMH) and the NRF-DAAD scholarship (TAE). MN received postdoctoral fellowships from the University of Cape Town (UCT) Health Sciences Faculty and UCT-Neuroscience Institute awards.

\section{Availability of data and materials}

The datasets during and/or analysed during the current study available from the corresponding author on reasonable request.

\section{Ethics approval and consent to participate}

The study was approved by the University of Cape Town Human Research Ethics Committee (HREC 257/2012) and all participants provided informed written consent

\section{Consent for publication}

All persons participating in this study agreed to their anonymous data being published and shared.

\section{Competing interests}

The authors declare that they have no competing interests.

\section{Author details}

${ }^{1}$ Neurology Research Group, Division of Neurology, Department of Medicine, Faculty of Health Sciences, E8-74, New Groote Schuur Hospital, University of Cape Town, Cape Town 7925, South Africa. ${ }^{2}$ UCT Neuroscience Institute, Cape Town, South Africa.

Received: 25 August 2020 Accepted: 27 November 2020

Published online: 11 December 2020

\section{References}

1. Heckmann JM, Nel M. A unique subphenotype of myasthenia gravis. Ann NY Acad Sci. 2018;1412:14-20.

2. Heckmann JM, Owen EP, Little F. Myasthenia gravis in South Africans: racial differences in clinical manifestations. Neuromuscul Disord. 2007:17:929-34.

3. Heckmann JM, Hansen P, Van Toorn R, Lubbe E, Janse van Rensburg E, Wilmshurst JM. The characteristics of juvenile myasthenia gravis among South Africans. S Afr Med J. 2012;102:532-6.

4. Europa TA, Nel M, Heckmann JM. Myasthenic ophthalmoparesis: time to resolution after initiating immune therapies. Muscle Nerve. 2018:58:542-9

5. Nel M, Jalali Sefid Dashti M, Gamieldien J, Heckmann JM. Exome sequencing identifies targets in the treatment-resistant ophthalmoplegic subphenotype of myasthenia gravis. Neuromuscul Disord. 2017;27:816-25.

6. Nel M, Mulder N, Europa TA, Heckmann JM. Using whole genome sequencing in an African subphenotype of myasthenia gravis to generate a pathogenetic hypothesis. Front Genet. 2019;10:136 
7. Nel M, Prince S, Heckmann JM. Profiling of patient-specific myocytes identifies altered gene expression in the ophthalmoplegic subphenotype of myasthenia gravis. Orphanet J Rare Dis. 2019;14:24.

8. Abecasis GR, Auton A, Brooks LD, DePristo MA, Durbin RM, Handsaker RE, Kang HM, Marth GT, McVean GA. An integrated map of genetic variation from 1,092 human genomes. Nature. 2012;491:56-65.

9. Fischer MD, Budak MT, Bakay M, Gorospe JR, Kjellgren D, Pedrosa-Domellof F, Hoffman EP, Khurana TS. Definition of the unique human extraocular muscle allotype by expression profiling. Physiol Genom. 2005:22:283-91.

10. Porter JD, Khanna S, Kaminski HJ, Rao JS, Merriam AP, Richmonds CR, Leahy P, Li J, Andrade FH. Extraocular muscle is defined by a fundamentally distinct gene expression profile. Proc Natl Acad Sci U S A. 2001;98:12062-7.

11. Porter JD, Israel S, Gong B, Merriam AP, Feuerman J, Khanna S, Kaminski HJ. Distinctive morphological and gene/protein expression signatures during myogenesis in novel cell lines from extraocular and hindlimb muscle. Physiol Genom. 2006;24:264-75.

12. Verma M, Fitzpatrick K, McLoon LK. Extraocular muscle repair and regeneration. Curr Ophthalmol Rep. 2017;5:207-15.

13. Cheng NC, Liao SL, Wang IJ, Lin IC, Tang YB. Fiber type and myosin heavy chain compositions of adult pretarsal orbicularis oculi muscle. J Mol Histol. 2007;38:177-82.

14. Porter JD, Baker RS. Muscles of a different "color": the unusual properties of the extraocular muscles may predispose or protect them in neurogenic and myogenic disease. Neurology. 1996;46:30-7

15. Goodmurphy CW, Ovalle WK. Morphological study of two human facial muscles: orbicularis oculi and corrugator supercilii. Clin Anat. 1999;12:1-11.

16. Kuang J, Yan X, Genders AJ, Granata C, Bishop DJ. An overview of technical considerations when using quantitative real-time PCR analysis of gene expression in human exercise research. PLoS ONE. 2018;13:30196438. https://doi.org/10.1371/journal.pone.0196438.

17. Maurer M, Bougoin S, Feferman T, Frenkian M, Bismuth J, Mouly V, et al. IL-6 and Akt are involved in muscular pathogenesis in myasthenia gravis. Acta Neuropathol Commun. 2015;3:1.

18. Kaminski HJ, Himuro K, Alshaikh J, Gong B, Cheng G, Kusner LL. Differential RNA expression profile of skeletal muscle induced by experimental autoimmune myasthenia gravis in rats. Front Physiol. 2016;7:524.

19. Rautenbach RM, Pillay K, Murray ADN, Heckmann JM. Extraocular muscle findings in myasthenia gravis associated treatment-resistant ophthalmoplegia. J Neuroophthalmol. 2017;37:414-7.

20. Andrade $\mathrm{FH}, \mathrm{McMullen} \mathrm{CA}$. Lactate is a metabolic substrate that sustains extraocular muscle function. Pflugers Arch. 2006;452:102-8.

21. Altick AL, Feng CY, Schlauch K, Johnson LA, von Bartheld CS. Differences in gene expression between strabismic and normal human extraocular muscles. Investig Ophthalmol Vis Sci. 2012;53:5168-77.

22. Agarwal AB, Feng CY, Altick AL, Quilici DR, Wen D, Johnson LA, von Bartheld CS. Altered protein composition and gene expression in strabismic human extraocular muscles and tendons. Investig Ophthalmol Vis Sci. 2016;57:5576-85.

23. Fischer MD, Gorospe JR, Felder E, Bogdanovich S, Pedrosa-Domellof F, Ahima RS, Rubinstein NA, Hoffman EP, Khurana TS. Expression profiling reveals metabolic and structural components of extraocular muscles. Physiol Genom. 2002;9:71-84.

24. Vandesompele J, De Preter K, Pattyn F, Poppe B, Van Roy N, De Paepe A, Speleman F. Accurate normalization of real-time quantitative RT-PCR data by geometric averaging of multiple internal control genes. Genome Biol. 2002;3:RESEARCH0034.

25. Pfaffl MW, Tichopad A, Prgomet C, Neuvians TP. Determination of stable housekeeping genes, differentially regulated target genes and sample integrity: BestKeeper-Excel-based tool using pair-wise correlations. Biotechnol Lett. 2004;26:509-15.

26. Schmittgen TD, Livak KJ. Analyzing real-time PCR data by the comparative C(T) method. Nat Protoc. 2008;3:1101-8.

27. Fukushima A. DiffCorr: an R package to analyze and visualize differential correlations in biological networks. Gene. 2013;518:209-14.

28. Park KA, Lim J, Sohn S, Oh SY. Myosin heavy chain isoform expression in human extraocular muscles: longitudinal variation and patterns of expression in global and orbital layers. Muscle Nerve. 2012;45:713-20.

29. Stupka N, Kintakas C, White JD, Fraser FW, Hanciu M, Aramaki-Hattori $\mathrm{N}$, et al. Versican processing by a disintegrin-like and metalloproteinase domain with thrombospondin-1 repeats proteinases-5 and -15 facilitates myoblast fusion. J Biol Chem. 2013;288:1907-17.

30. Beckmann JS, Spencer M. Calpain 3, the "gatekeeper" of proper sarcomere assembly, turnover and maintenance. Neuromuscul Disord. 2008;18:913-21.

31. Coulombe P, Rodier G, Pelletier S, Pellerin J, Meloche S. Rapid turnover of extracellular signal-regulated kinase 3 by the ubiquitin-proteasome pathway defines a novel paradigm of mitogen-activated protein kinase regulation during cellular differentiation. Mol Cell Biol. 2003;23:4542-58.

32. Tryon LD, Vainshtein A, Memme JM, Crilly MJ, Hood DA. Recent advances in mitochondrial turnover during chronic muscle disuse. Integr Med Res. 2014:3:161-71.

33. van Dam S, Vosa U, van der Graaf A, Franke L, de Magalhaes JP. Gene co-expression analysis for functional classification and gene-disease predictions. Brief Bioinform. 2018;19:575-92.

34. Dardenne E, Polay Espinoza M, Fattet L, Germann S, Lambert MP, Neil H, et al. RNA helicases DDX5 and DDX17 dynamically orchestrate transcription, miRNA, and splicing programs in cell differentiation. Cell Rep. 2014;7:1900-13.

35. Chang W, Gelman MS, Prives JM. Calnexin-dependent enhancement of nicotinic acetylcholine receptor assembly and surface expression. J Biol Chem. 1997;272:28925-32.

36. Wang L, Yan Z, Vihinen H, Eriksson O, Wang W, Soliymani R, et al. FAM92A1 is a BAR domain protein required for mitochondrial ultrastructure and function. J Cell Biol. 2019;218:97-111.

37. McGourty CA, Akopian D, Walsh C, Gorur A, Werner A, Schekman R, et al. Regulation of the CUL3 ubiquitin ligase by a calcium-dependent Coadaptor. Cell. 2016;167:525-38.

38. Chou CH, Shrestha S, Yang CD, Chang NW, Lin YL, Liao KW, et al. miRTarBase update 2018: a resource for experimentally validated microRNAtarget interactions. Nucleic Acids Res. 2018;46:D296-302.

39. Zeiger U, Khurana TS. Distinctive patterns of microRNA expression in extraocular muscles. Physiol Genom. 2010;41:289-96.

40. Cisternas P, Henriquez JP, Brandan E, Inestrosa NC. Wnt signaling in skeletal muscle dynamics: myogenesis, neuromuscular synapse and fibrosis Mol Neurobiol. 2014:49:574-89.

41. Summermatter S, Bouzan A, Pierrel E, Melly S, Stauffer D, Gutzwiller S, et al. Blockade of metallothioneins 1 and 2 increases skeletal muscle mass and strength. Mol Cell Biol. 2017;37:e00305-e316.

42. Munoz-Canoves P, Scheele C, Pedersen BK, Serrano AL. Interleukin-6 myokine signaling in skeletal muscle: a double-edged sword? Febs J. 2013;280:4131-48.

43. Consortium GT. The genotype-tissue expression (GTEx) project. Nat Genet. 2013;45:580-5.

44. Hong Y, Liang X, Gilhus NE. AChR antibodies show a complex interaction with human skeletal muscle cells in a transcriptomic study. Sci Rep. 2020;10:11230.

45. Europa TA, Nel M, Heckmann JM. A review of the histopathological findings in myasthenia gravis: clues to the pathogenesis of treatmentresistance in extraocular muscles. Neuromuscul Disord. 2019;29:381-7.

46. Sartori R, Gregorevic P, Sandri M. TGFbeta and BMP signaling in skeletal muscle: potential significance for muscle-related disease. Trends Endocrinol Metab. 2014:25:464-71.

47. Glass DJ. PI3 kinase regulation of skeletal muscle hypertrophy and atrophy. Curr Top Microbiol Immunol. 2010;346:267-78.

48. Auret J, Abrahams A, Prince S, Heckmann JM. The effects of prednisone and steroid-sparing agents on decay accelerating factor (CD55) expression: implications in myasthenia gravis. Neuromuscul Disord. 2014;24:499-508.

49. Nel M, Buys JM, Rautenbach R, Mowla S, Prince S, Heckmann JM. The African-387 C>TTGFB1 variant is functional and associates with the ophthalmoplegic complication in juvenile myasthenia gravis. J Hum Genet. 2016;61:307-16.

50. McLoon LK, Wirtschafter J. Activated satellite cells in extraocular muscles of normal adult monkeys and humans. Investig Ophthalmol Vis Sci. 2003:44:1927-32.

51. Anderson BC, Christiansen SP, McLoon LK. Myogenic growth factors can decrease extraocular muscle force generation: a potential biological approach to the treatment of strabismus. Investig Ophthalmol Vis Sci. 2008:49:221-9 
52. McLoon LK, Harandi VM, Brannstrom T, Andersen PM, Liu JX. Wnt and extraocular muscle sparing in amyotrophic lateral sclerosis. Investig Ophthalmol Vis Sci. 2014;55:5482-96.

53. Zhou Y, Kaminski HJ, Gong B, Cheng G, Feuerman JM, Kusner L. RNA expression analysis of passive transfer myasthenia supports extraocular muscle as a unique immunological environment. Invest Ophthalmol Vis Sci. 2014:55:4348-59.

54. Sekulic-Jablanovic M, Ullrich ND, Goldblum D, Palmowski-Wolfe A, Zorzato F, Treves S. Functional characterization of orbicularis oculi and extraocular muscles. J Gen Physiol. 2016;147:395-406.

\section{Publisher's Note}

Springer Nature remains neutral with regard to jurisdictional claims in published maps and institutional affiliations.
Ready to submit your research? Choose BMC and benefit from:

- fast, convenient online submission

- thorough peer review by experienced researchers in your field

- rapid publication on acceptance

- support for research data, including large and complex data types

- gold Open Access which fosters wider collaboration and increased citations

- maximum visibility for your research: over $100 \mathrm{M}$ website views per year

At BMC, research is always in progress.

Learn more biomedcentral.com/submissions 